VENTURINI et al., v(5), n5, p. 950 - 959, 2012.

Revista Eletrônica em Gestão, Educação e Tecnologia Ambiental

REGET/UFSM (e-ISSN: 2236-1170).

\title{
ESTUdo dA SECAGEM E EXTRAÇÃo De SEMENTES DE MAMÃo (Carica Papaya L.)
}

\author{
Taís Venturini ${ }^{1}$, Lilian R. Benchimol ${ }^{1}$, Daniel A. Bertuol ${ }^{1}$, Marcelo B. da Rosa ${ }^{2}$, Lucas Meili ${ }^{{ }^{*}}$ \\ tais.venturini@gmail.com, righi.lilian@gmail.com, dbertuol@gmail.com, marcelobdarosa@gmail.com, \\ *Autor para correspondência: lucasmeili@ufsm.br \\ ${ }^{1}$ Departamento de Engenharia Química (DEQ-CT/UFSM). \\ ${ }^{2}$ Laboratório de Análises Químicas - LACHEM (DQ-CCNE/UFSM). \\ Av. Roraima no 1000 - Cidade Universitária - Bairro Camobi \\ Santa Maria - RS - CEP: 97105-900 - Fone: +55-3220-8607
}

\section{RESUMO}

Em virtude de características como o alto teor em óleo, quantidade expressivas de glucosinolatos, fonte de pectina, entre outros, buscou-se através desse trabalho avaliar as melhores condições de secagem e extração do óleo das sementes de mamão do tipo Caricapapaya L. Para o desenvolvimento deste, as sementes foram secas em estufa de esterilização nas temperaturas de 60 e $70^{\circ} \mathrm{C}$ e, em seguida, foram realizadas extrações via sonicação de amostras com diâmetros médio de $1,200 \mathrm{~mm}, 0,780 \mathrm{~mm}$ e inferior a $0,655 \mathrm{~mm}$. Com os dados obtidos, curvas de secagem foram construídas e ajustadas segundo modelos matemáticos. Espectros de absorbância em função do comprimento de onda também foram construídos e analisados.

\section{INTRODUÇÃO}

O mamão (Carica papaya) é uma planta muito comum em regiões de clima tropical e subtropical. Foi descoberto na região entre o sul do México e o norte da Nicarágua e introduzido no Brasil, em 1587, devido ao clima favorável. De acordo com Melo (2010), o Brasil é um dos principais produtores mundiais junto com o México, Nicarágua, entre outros países.

A fruta é consumida tanto in natura, como também processada na forma de geléia, doces e polpa e com isso, uma porcentagem significativa de resíduos são gerados. Para o processamento da fruta são retiradas casca e sementes, o que constituí cerca de $50 \%$ da mesma. Isso pode acarretar problemas ambientais devido ao resíduo produzido. Quanto maior o tamanho do fruto, maiores quantidades de sementes este contêm e, conforme Allan (1969), um único mamão pode produzir em torno de 1000 sementes ou até mais.

Inúmeros trabalhos têm demonstrado que o extrato de semente de mamão, obtido por extração aquosa ou com solventes orgânicos, apresenta um componente bioativo, o isotiocianato de benzila (Dar et al., 1965; Ettlinger and Hodgkins, 1956; Krishnakumari and Majumder, 1960; Tang, 1971). Os isotiocianatos são obtidos a partir da conversão dos glicosinolatos pela ação da enzima mirosinase, a qual está presente no próprio vegetal ou na microflora do trato digestivo humano, esta enzima inicia sua atividade pela injúria ou ação de fungos no tecido vegetal das plantas frescas (Bones; 1996). Os glicosinolatos (ß-tioglicosídio-N-hidroxissulfatos) ocorrem em 16 famílias de espécies vegetais, existindo, pelo menos, 120 estruturas diferentes identificadas em plantas (Castro et al.; 2008). Diversos estudos demonstram que os vegetais possuem propriedades químio-protetoras, as 


\section{Revista Eletrônica em Gestão, Educação e Tecnologia Ambiental REGET/UFSM (e-ISSN: 2236-1170).}

quais são atribuídas à ocorrência em baixas concentrações de certas substâncias como os glicosinolatos. $O$ isotiocianato de benzila, formado a partir dos glicosinolatos, encontrado no mamão, tem ação inibidora no desenvolvimento de câncer de pâncreas (Kermanshai et al.; 2001) e sobre o câncer de pulmão, aumentando a ocorrência de apoteoses das células cancerosas (Kuang e Chen; 2004; D’agostini et al.; 2005). Além destas características, estudos laboratoriais confirmaram que várias preparações de sementes de mamão papaya podem matar, de forma efetiva, helmintos in vitro e in vivo (Robinson; 1958; Krishnakumari and Majumder, 1960; Dar et al., 1965; Lal et al.,1976; Kermanshahi; 2001), sendo o benzil isotiocianato o composto ativo responsável por tal característica.

Em relação à conservação de materiais biológicos, a maioria perde a validade em curtos períodos de armazenamento (Aroeira, 1962; Madhava-Rao e Shanmugavelu, 1971; Yahiro, 1979 e J.R. Viggiano et al.). Desta forma, é necessário considerar seu processo de deterioração, com o intuito de conservar e armazenar o material da melhor maneira possível. Este processo depende de fatores como o grau de umidade, condições do ambiente de armazenamento, temperatura e umidade relativa do ar, atividade enzimática, longevidade da espécie, qualidade inicial das sementes e do recipiente de armazenamento (Carvalho e Nakagawa, 2000). No entanto, as principais variáveis que afetam o estado de conservação das sementes são a temperatura, afetando a velocidade das reações de decomposição, e a umidade relativa do ar, dada a sua relação com a quantidade de água presente nas sementes (Delouche et al., 1973). Assim, a validade da semente pode ser aumentada com a redução do teor de água através da operação de secagem e com alterações nas condições do ambiente de armazenamento.

A secagem é o processo de eliminação de líquidos sobre a superfície ou interior de um material através da evaporação e transferência de calor e massa. É utilizado para impedir a decomposição de materiais biológicos, bem como facilitar o transporte e armazenamento das mesmas. (Nonhebel e Moss, 1971; Keey, 1978; Strumillo e Kudra, 1986). A pressão de vapor de água, a temperatura, a velocidade do ar e da difusão da água e as espessuras da superfície são os fatores que determinam a secagem, ou seja, regem os fenômenos de transporte envolvidos (Nonhebel e Moss, 1971; Strumillo e Kudra,1986). A realização de experimentos de secagem permite verificar a influência desses fatores para determinadas condições de processo (Costa, 1999). Na literatura especializada são encontrados diversos métodos teóricos, empíricos e semiempíricos para a análise da secagem de produtos higroscópicos. Os métodos teóricos, em geral, consideram as condições, os mecanismos internos de transferência de energia e massa, incluindo seus efeitos, e as condições operacionais externas (Brooker et al., 1992). Os métodos empíricos têm como base uma abordagem experimental onde apresentam uma relação direta entre o tempo de secagem e o conteúdo médio de umidade. Nesses modelos são omitidos parâmetros físicos relacionados ao processo de secagem, sendo os parâmetros dos modelos somente utilizados para o ajuste dos dados, não descrevendo de forma fundamental os processos importantes durante a operação, entretanto são mais fáceis de utilizar que os modelos teóricos (Keey, 1972). Já os modelos semi-empíricos mesclam a teoria relacionada ao processo e a facilidade de utilização. Segundo Brooker et al. (1992) os modelos semi-empíricos se baseiam na Lei de resfriamento de Newton aplicada à transferência de massa, onde assume-se que a resistência à transferência de massa ocorra apenas na superfície do sólido e que a operação ocorre em condições isotérmicas.

Ao trabalhar com produtos naturais, a extração dos princípios ativos da massa vegetal, vem a ser uma etapa crucial para a obtenção de um produto final com elevados teores de substâncias ativas. A escolha do método extrativo e do solvente ou mistura de solventes adequados deve seguir critérios rígidos visando uma metodologia eficiente e de baixo custo operacional. A extração de princípios 


\section{Revista Eletrônica em Gestão, Educação e Tecnologia Ambiental REGET/UFSM (e-ISSN: 2236-1170).}

ativos pode ser realizada por inúmeros métodos extrativos que podem ser classificados em métodos clássicos, como a maceração e a percolação, e métodos mais modernos, como extração assistida por ultra-som, fluido supercrítico e extratores com controle de temperatura e agitação (Thoma et al; 2001).

Os fundamentos para aplicação do ultrasonicação em processos de extração estão relacionados com as ondas de choque resultantes da aplicação do campo acústico sobre um meio material. Essas ondas acentuam a interação entre o solvente e a superfície dos sólidos, aumentando, na solução, a concentração das espécies presentes no material sob investigação. Dentre as vantagens do uso de ultrasom, a redução do tempo de reação, de consumo de reagentes, seletividade, favorecimento de reações, simplificação de sistemas reacionais e redução de custos são as principais que podem ser citadas.

A partir do exposto anteriormente, este trabalho teve como objetivo analisar a secagem em camada fina em duas temperaturas (60 e 70 C) avaliando qual modelo de secagem (Lewis, Page, Midilli, Henderson e Pabis) é capaz de representar de forma satisfatória os dados experimentais. Além disso, foram analisadas algumas variáveis importantes na operação de extração via sonificação com etanol a 40 ำ: diâmetro da partícula $(1,2,0,78$ e inferior a 0,65mm), tempo de extração $(0-240 \mathrm{~min})$ e temperatura de secagem.

\section{METODOLOGIA}

Inicialmente, as sementes do mamão passaram por um processo de remoção das membranas externas chamadas de sarcotesta através do atrito da semente com uma esponja de aço em uma superfície rígida. Então, tréplicas de amostras contendo a mistura de sementes e sarcotestas foram postas na estufa (Brasdonto, modelo 5) à temperatura de $105^{\circ} \mathrm{C}$ para verificação da umidade que se encontra em torno de $81,34 \%$. Sequencialmente, foram realizadas as secagens à 60 e $70^{\circ} \mathrm{C}$ em triplicata na mesma estufa anteriormente citada. Em intervalos pré-determinados as amostras eram retiradas para pesagens até manter-se a massa constante. Com os dados obtidos, as curvas de secagem foram calculadas e ajustadas segundo modelos matemáticos.

Para dar início ao processo de extração, as sementes de mamão previamente secas foram moídas em moinho de sapatas (Marconi modelo MA 880) por 40 minutos. Posteriormente, foi realizada uma separação por ensaio de peneiras da série Tyler com agitador Produtest. 0 material previamente separado foi divido em frações de $1,200 \mathrm{~mm}, 0,780 \mathrm{~mm}$ e inferior a $0,655 \mathrm{~mm}$. Para cada fração foram pesadas amostras de $0,1 \mathrm{~g}$ para os ensaios de extração. Cada amostra foi inserida em diferentes tubos de ensaio, no qual se adicionou $10 \mathrm{~mL}$ do solvente, etanol $92,8 \%$. Os tubos de ensaio foram termostatizados e sonicados (Ultrasom Bandelin Sonorex Super modelo RK510H) durante 240 minutos, sendo alíquotas retiradas em intervalos de 30 minutos.

Os espectros de absorção molecular foram obtidos entre 200 e $700 \mathrm{~nm}$. As medidas foram realizadas em um espectrofotômetro com arranjo de diodos HP 8453 (Hewlett Packard), utilizando uma célula de quartzo de $10 \mathrm{~mm}$ de espessura do caminho óptico. 


\section{Revista Eletrônica em Gestão, Educação e Tecnologia Ambiental REGET/UFSM (e-ISSN: 2236-1170).}

\section{Considerações teóricas sobre os modelos de cinética de secagem}

Para tentar descrever com precisão o comportamento da secagem, os dados de secagem obtidos nas duas temperaturas estudadas foram ajustados a 04 modelos empíricos e semiempíricos mais comuns para secagem em camada delgada, apresentados na Tabela 1 . Nos modelos apresentados, t é o tempo de secagem e RU representa a razão adimensional de umidade dada pela seguinte equação:

$$
R U=\frac{\left(M-M_{g}\right)}{\left(M_{0}-M_{g}\right)}
$$

onde, $M$ é o conteúdo de umidade do produto, $M_{0}$ é o conteúdo de umidade do produto no início e $M_{e}$ é a umidade de equilíbrio. Como os valores de $M_{e}$ são relativamente muito pequenos quando comparados com $\mathrm{M}$ e $\mathrm{M}_{0}$, então:

$R U=\frac{M}{M_{0}}(2)$

A qualidade de representação dos dados experimentais pelos modelos testados foi avaliada através do coeficiente de correlação $\left(R^{2}\right)$ e pelo parâmetro chi-quadrado $\left(\chi^{2}\right)$. Quanto maiores os valores de $R^{2}$ e menores os valores de $\chi^{2}$ melhor o ajuste obtido pelos modelos. $O \chi^{2}$ pode ser obtido por meio da equação que se segue:

$\chi^{2}=\frac{\sum_{i=1}^{N}\left(R U_{e x p, i}-k U_{\text {pred }, i}\right)^{2}}{N-z}(3)$

onde $R U_{\text {exp,i }}$ e $R U_{\text {pred,i }}$ são o i valor da razão adimensional de umidade experimental e predita, respectivamente, $\mathrm{N}$ é o número de observações e $\mathrm{z}$ é o número de parâmetros do modelo. Para o estudo dos modelos foi utilizada uma análise de regressão não linear com o auxílio do software Statistica 7.0.

Tabela 1: Modelos empíricos e semi-empíricos utilizados

\begin{tabular}{|c|c|c|}
\hline $\begin{array}{c}\text { Número do } \\
\text { Modelo }\end{array}$ & Modelo & $R U=\exp \left(-k \cdot t^{n}\right)$ \\
\hline 1 & $\begin{array}{c}\text { Lewis (Lewis; 1921 } \\
\text { apudBarrozo; 1995) }\end{array}$ & $R U=\exp \left(-k \cdot t^{\text {n }}\right)$ \\
\hline 2 & $\begin{array}{c}\text { Page (Page; 1949; } \\
\text { apudBarrozo; 1995) }\end{array}$ & $R U=a \cdot \exp (-k \cdot t)$ \\
\hline 3 & $\begin{array}{c}\text { Henderson e Pabis (Brooker et } \\
\text { al. 1979 apudBarrozo; 1995) }\end{array}$ & $R U=a \cdot \exp \left(-k \cdot t^{\text {n }}\right)+b \cdot t$ \\
\hline 4 & $\begin{array}{c}\text { Midilli (Midilliet al. } \\
\text { 2002) }\end{array}$ & \\
\hline
\end{tabular}




\section{Revista Eletrônica em Gestão, Educação e Tecnologia Ambiental REGET/UFSM (e-ISSN: 2236-1170).}

\section{RESULTADOS}

\section{Ensaios de Secagem}

Os dados experimentais obtidos foram representados em termos de conteúdo de umidade em base seca. Esses dados de umidade a qualquer momento do processo foram convertidos em razão adimensional de umidade nas temperaturas analisadas. Os modelos de secagem em camada fina foram comparados em termos estatísticos por meio dos parâmetros $R^{2}$ e $\chi^{2}$. Na Tabela 2 estão apresentados os resultados estatísticos para os modelos analisados onde, de acordo com o critério de comparação. Os modelos 2 e 4 obtiveram os maiores valores de $\mathrm{R}^{2}$ e os menores valores de $\chi^{2}$.

Tabela 2: Resultados estatísticos para os modelos analisados

\begin{tabular}{|c|c|c|c|c|}
\cline { 2 - 5 } \multicolumn{1}{c|}{} & \multicolumn{4}{c|}{ Teste Estatístico } \\
\cline { 2 - 5 } \multicolumn{1}{c|}{} & $\mathbf{R}^{\mathbf{2}} \mathbf{C}$ & $\mathbf{R}^{\mathbf{2}}$ & $\mathbf{\chi}^{\mathbf{}} \mathbf{C}$ \\
\hline Modelos & 0,99041 & 0,002011 & 0,98747 & 0,002724426 \\
\hline 1 & 0,99565 & 0,000935 & 0,99972 & $<0,0001$ \\
\hline 2 & 0,99052 & 0,002032 & 0,98925 & 0,00240854 \\
\hline 3 & 0,9967 & 0,000742 & 0,99974 & $<0,0001$ \\
\hline 4 & &
\end{tabular}

O modelo 4 tem mais parâmetros ajustáveis e apresenta performance similar à obtida no modelo 2, desta forma, o modelo 2 foi o escolhido para representar os dados experimentais. Os valores dos parâmetros estimados pelo modelo para as duas temperaturas testadas estão apresentados na Tabela 3. A boa concordância entre os dados experimentais e os preditos pelo modelo podem ser melhor observados na Figura 1. Observa-se nesta figura um comportamento cinético típico, com um período de taxa de secagem constante (até 150 min para 60 $\mathrm{C}$ e até 90 min) e um período de taxa decrescente. Além disso, pode-se notar claramente, nesta figura, que a temperatura exerceu influência significativa na cinética de secagem. Enquanto que para 60 ㅇ $C$ são necessários 300 min para que se atinja o equilíbrio da operação, a 70 C este equilíbrio é alcançado em menos de $200 \mathrm{~min}$.

Tabela 3: Parâmetros estimados pelo Modelo 2.

\begin{tabular}{|l|l|l|}
\cline { 2 - 3 } \multicolumn{1}{c|}{} & \multicolumn{2}{|c|}{ Parâmetros } \\
\hline Temperaturas & K & $\mathbf{n}$ \\
\hline 60 & 0,001857 & 1,3487 \\
\hline 70 & 0,001473 & 1,5369 \\
\hline
\end{tabular}




\section{Revista Eletrônica em Gestão, Educação e Tecnologia Ambiental REGET/UFSM (e-ISSN: 2236-1170).}

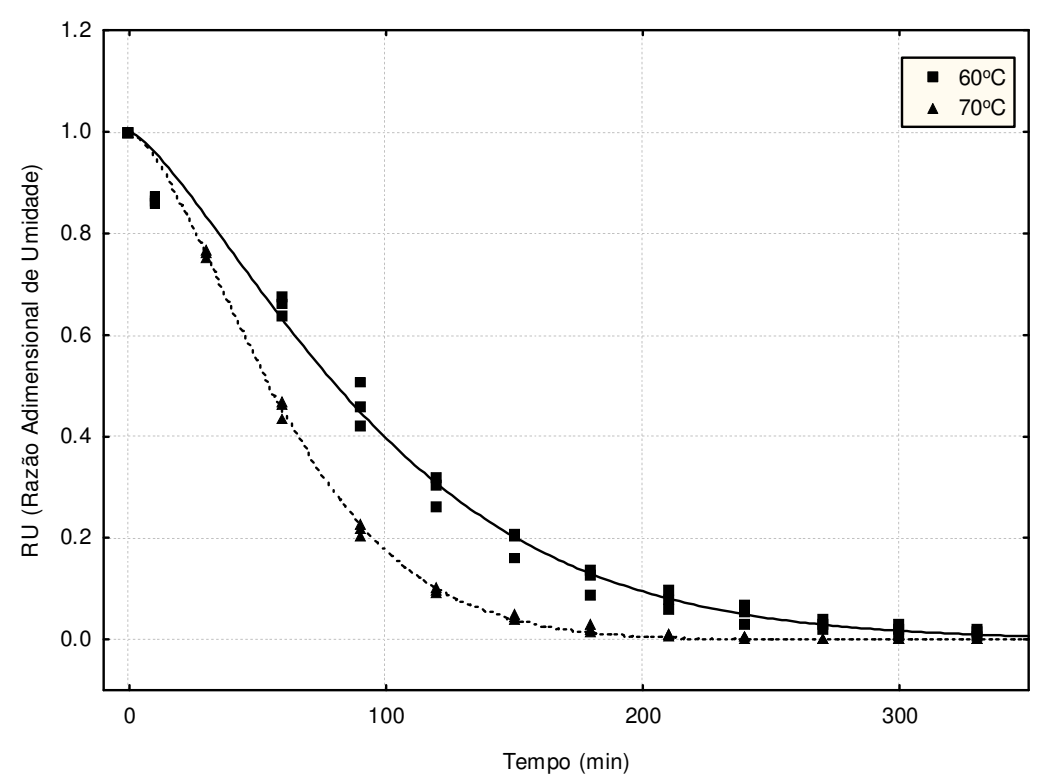

Figura 1: Razão adimensional de umidade em função do tempo para as temperaturas de 60 e 70 은 C com o ajuste obtido pelo modelo 2 .

\section{Ensaios de Extração}

Para o processo de extração, os diâmetros foram previamente testados e analisados espectrofotometricamente. Antes, as amostras extraídas foram filtradas e logo a seguir analisadas.

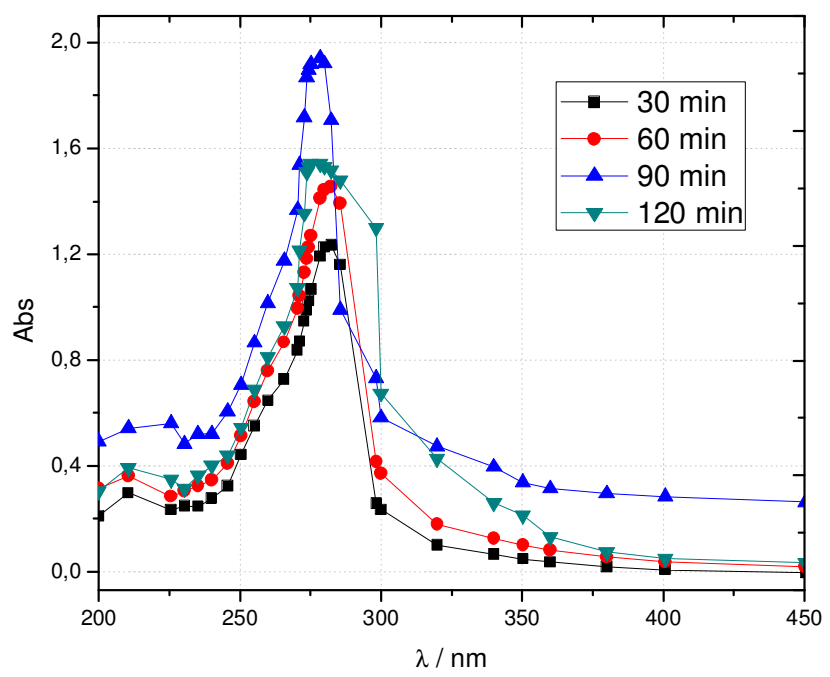

Figura 3: Espectros para partículas com diâmetro médio menor que $1,200 \mathrm{~mm}$ seca a $70 \cong \mathrm{C}$ 


\section{Revista Eletrônica em Gestão, Educação e Tecnologia Ambiental REGET/UFSM (e-ISSN: 2236-1170).}

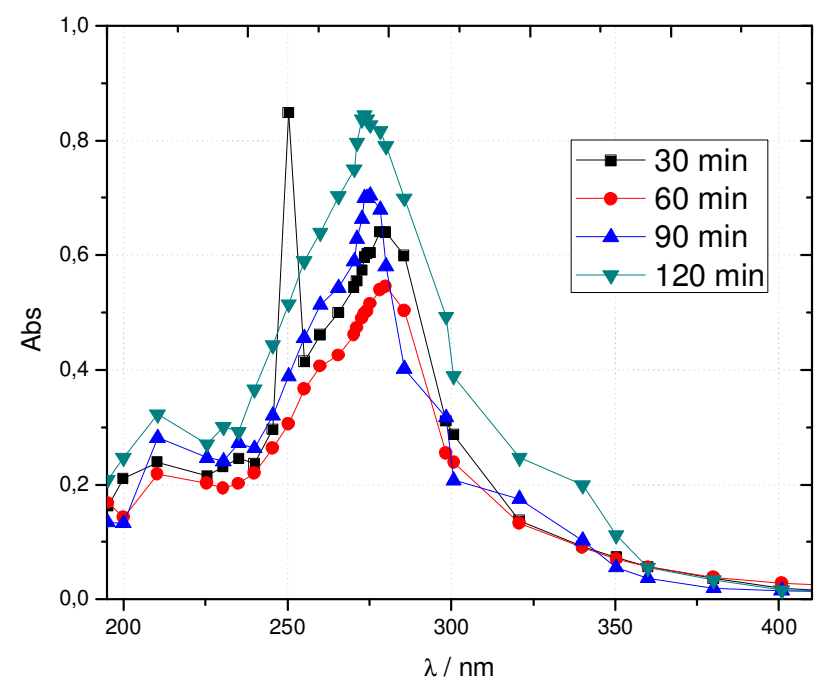

Figura 4: Espectros para partículas com diâmetro médio menor que $0,780 \mathrm{~mm}$ seca a 70 C

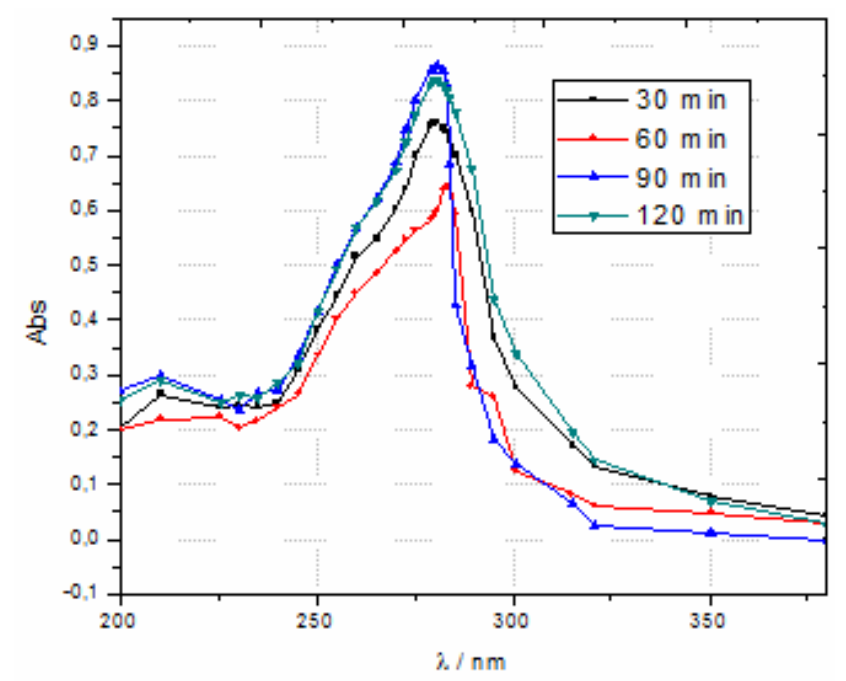

Figura 5: Espectros para partículas com diâmetro médio menor que $0,655 \mathrm{~mm}$ seca a 70 ㅇ C

De acordo com a comparação entre os espectros obtidos, o menor diâmetro de partículas mostrou-se mais eficiente no procedimento de extração devido a sua maior área de contato com o solvente. 


\section{Revista Eletrônica em Gestão, Educação e Tecnologia Ambiental REGET/UFSM (e-ISSN: 2236-1170).}

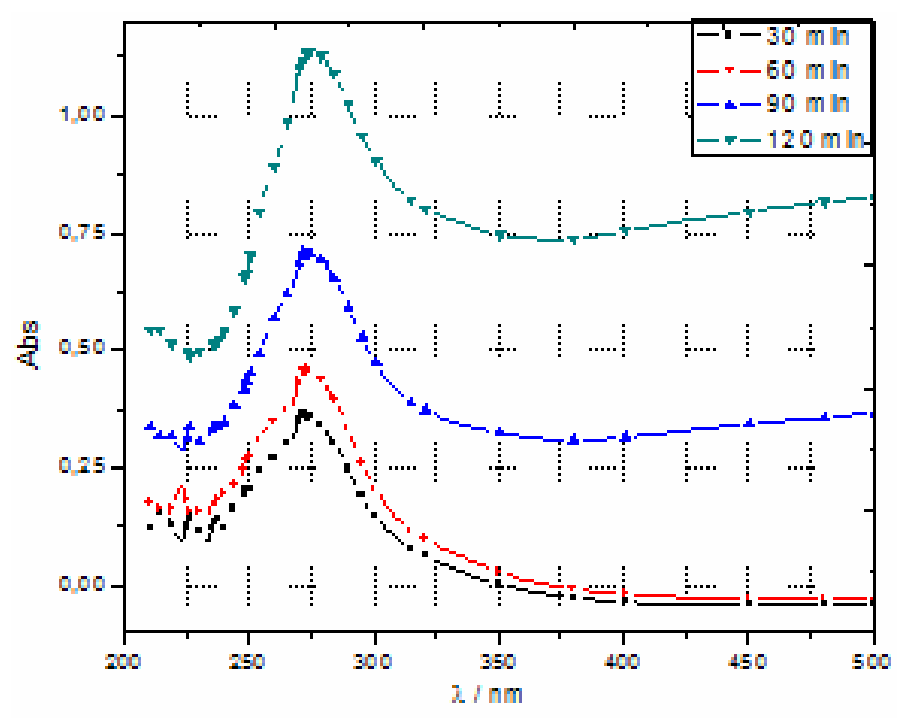

Figura 6: Espectros para partículas com diâmetro médio 0,655mm seca a 60ㅇ C

Através dos resultados espectrofotométricos, observou-se a influência do tempo e do diâmetro de partícula para a extração, como mostra a Figura 5 e 6, em que o menor diâmetro exposto ao solvente por um maior período mostrou maior eficácia de extração. O comprimento de onda considerado para monitoramento foi de $275 \mathrm{~nm}$, onde o aumento de seu valor de absorvância foi acompanhado.

\section{CONCLUSÃO}

A partir dos resultados obtidos pode-se afirmar que a cinética de secagem obteve um comportamento típico com períodos de taxa constante e decrescentes bem definidos. Dos modelos matemáticos testados, o de Page foi o que se mostrou mais adequado para representar os dados experimentais. Estes resultados preliminares de secagem servirão como base para novos experimentos em secadores de túnel de vento e de leitos móveis.

Os dados espectrofotométricos comprovam a influência do diâmetro de partícula para a eficiência do processo, assim como, o tempo exposto ao solvente e a temperatura para acelerar a extração.

A ultrasonicação mostrou-se adequada e potencialmente utilizável em estudos que procuram otimizar processos de extração tanto a indústria alimentícia, quanto farmacêutica e da fitomedicina.

Assim, o presente trabalho traz contribuições fundamentais aos processos de otimização de processos industrais e será usado como subsídio prático-teórico para modelagem matemática de extrações e obtenção de perfis de secagem mais bem definidos e otimizados. 


\section{Revista Eletrônica em Gestão, Educação e Tecnologia Ambiental REGET/UFSM (e-ISSN: 2236-1170).}

\section{AGRADECIMENTOS}

Os autores agradecem à Fundação de Amparo à Pesquisa do Estado do Rio Grande do Sul (FAPERGS-11/1724-9) e ao Conselho Nacional de Desenvolvimento Científico e Tecnológico (CNPq).

\section{REFERÊNCIAS}

Barrozo, M. A. S. (1995) Transferência de calor e massa entre o ar e sementes de soja em leito deslizante e escoamentos cruzados. UFSCar, São Carlos-SP (Tese).

Batista, L. M. (2004). Secagem de quitosana obtida a partir de resíduos de camarão: análise da cinética de secagem considerando encolhimento. Fundação Universidade do Rio Grande (dissertação).

Bones, A. M.; Rossiter, B. (1996). Physiol. Plant. 97, 1994.

Braga, N.P (2002). Influência da secagem no rendimento e na composição do óleo essencial de folhas de Eucalyptuscitroa. Universidade Estadual de Campinas (dissertação).

Brooker, D. B.; Baker-Arkema, F. W.; Hall, C. W. Drying and storage of grains and oilseeds. New York: AVI Book, 1992. 450p.

Castro, I. M.; Anjos, M. R.; Oliveira, E.S. (2008). Determinação de isotiocianato de benzila em Caricapapaya utilizando cromatografia gasosa com detectores seletivos. Quim. Nova, Vol. 31, No. 8, 1953-1959.

Corrêa, R. M.; Bertolucci, S. K. V.; Pinto, J. E. B. P.; Reis, E. S.; Alves, T. L. (2004). Rendimento de óleo essencial e caracterização organoléptica de folhas de assa-peixe submetidas a diferentes métodos de secagem. Ciênc. Agrotec., Lavras, v.28, no 2, p.341-346.

Dar, R. N., Garg, L.C., Pathak, R.D. (1965). Anthelmintic activity of Carica Papaya seeds. The Indian Journal of Pharm.;27:335-6.

D’agostini, F.; Izzotti, A.; Balansky, R. M.; Bennicelli, C.; De Flora, S. (2005); Mutat. Res. 591, 173.

Estanislau, W.T. (2007). Conservação de sementes de mamão em função da presença da sarcotesta, teor de água das sementes e tipo de embalagem. Universidade Federal de Viçosa (tese).

Ettlinger, M.G., Hodgkins, J.E.(1956). The mustard oil of papaya seed.Journal of Organic Chemistry 21, 204205.FARIAS, M. R. (2003). Em Avaliação da qualidade de matérias-primas vegetais; Simões, C. M. O.;Schenkel, E. P.; Gosmann, G.; Mello, J. C. P.; Mentz, L. A.; Petrovick, P. R., eds.; Eds. UFRGS - UFSC: Rio Grande do Sul - Santa Catarina.

Food and Agriculture Organization of the United Nations (FAOSTATS).Statistical Databases. Agriculture.2010. <http:// www.fao.org/faostat>

Freitas, L.A.P; Freire, J.T. (2001) Heat transfer in a draft tube spouted bed with bottom solids feed, Powder Technology, v. 114, p. 152-162, 2001.

Kermanshai, R.; McCarry, B. E.; Rosenfeld, J.; Summers, P. S.; Weretilnyk, E. A., Sorger, G. J. (2001). Benzyl isothiocyanate is the chief or sole anthelmintic in papaya seed extracts. Phytochemistry 57, 427-435. 


\section{Revista Eletrônica em Gestão, Educação e Tecnologia Ambiental REGET/UFSM (e-ISSN: 2236-1170).}

Krishnakumari, M. K.; Majumder, S. K. (1960). Studies on the anthelmintic action of seeds of Carica papaya Linn.. Ann. Biochem. Exp. Med.,2:551-6.

Kuang, Y. F.; Chen, Y. H. (2004). Food Chem. Toxicol., 42, 1711.

Kudra, T.; Mujumdar, A.S. (2002). Advanced Drying Technologies, Marcel Dekker, New York.

Lal J.;Chandra S.; Raviprakash V.; Sabir M. (1976). In vitro anthelmintic action of some indigenous medicinal plants on Ascaridiagalli worms. Indian Journ of Physiol and Pharmacol;20:64-8.

Matherson, B. et al. (1996). Effect on serum lipids of monoinsatured oil and margarine in the diet of an Antartic Expedition.The American Journal of Clinical Nutrition, v. 63, p. $993-941$.

Mathur, K.B.; Epstein, N. (1974). Spouted Bed, Academic Press, New York.

McCormick, P. Y. (1983). Solids Drying Fundamentals. In: Perry, J.; Chilton, C. H.. Chemical Engineering Handbook, McGraw Hill Co., sec. 20-24.

Melo, M. L. S. (2010). Caracterização, estabilidade oxidativa e determinação do potencial energético do biodiesel derivado do mamão (caricapapaya L.): uma fonte não convencional. Universidade Federal da Paraíba, João Pessoa, 151 p. (tese).

Moretto, E.;Fett, R. (1989). Tecnologia de óleos e gorduras vegetais. Rio de Janeiro: Varela.

Midilli, A.; Kucuk, H.; Yapar, Z. (2002) A new model for single-layer drying.Dryingtechnology, 20, 1503-1513.

Mujumdar, A. S. (2006). Handbookof Industrial Drying. 3. ed. Taylor \& Francis Group.

Paques, F.W; Macedo, G.A. (2006). Lipases de látex vegetais: propriedades e aplicações industriais. Química Nova, v. 29, n.1, p. $93-99$.

Revista Brasileira de Fruticultura, v. 15, n. 2, p.147-151, 1993.

Revista Brasileira de Sementes, vol. 22, no 2, p.279-287, 2000.

Revista Brasileira de Fruticultura, v. 32, n. 3 p. 657-959.

Robinson P. (1958)Seeds of Carica papaya for mass treatment against Ascariasis. Indian Journ of Child Health;7:815-7.

Serrano, L. A. L.; Cattaneo, L. F. (2010). O CULTIVO DO MAMOEIRO NO BRASIL.

Silva, F.; Casalli, V. W. D. (2000). Plantas medicinais e aromáticas: Pós-colheita e óleos essenciais, Arte e Livros: Minas Gerais.

Tang, C.S. (1971). Benzyl Isothiocyanate of Papaya Fruit. Phytochem;10:117-21.

Thoma, M., Vinatoru, M., Paniwnyk, L. e Mason, T.J., Investigation of the Effects of Ultrasound on Vegetal Tissues During Solvent Extraction, Ultrasonics Sonochemistry, v.8, p. 137-142, 2001. 